\title{
Glioma and glioblastoma - how much do we (not) know? (Review)
}

\author{
IVANA JOVČEVSKA, NINA KOČEVAR and RADOVAN KOMEL \\ Medical Center for Molecular Biology, Institute of Biochemistry, Faculty of Medicine, \\ University of Ljubljana, Ljubljana 1000, Slovenia
}

Received April 4, 2013; Accepted August 2, 2013

DOI: $10.3892 / \operatorname{mco} .2013 .172$

\begin{abstract}
Cancer is a heterogeneous disease, which provides a broad field for investigation, while simultaneously reducing the chances for a universal treatment. Malignant gliomas are the most common type of primary brain tumors. The heterogeneity of gliomas regarding clinical presentation, pathology and response to treatment makes this type of tumor a challenging area of research. As the clinical symptoms may be unspecific (e.g., seizures and headaches) it is often difficult to diagnose a patient in the early stages of the disease. Thus far, there are no known genetic patterns of inheritance of this disease. Currently, the treatment of glioblastoma involves surgery, whenever possible, followed by radiation and chemotherapy. Experimental procedures, such as passive and active immunotherapy, use of angiogenesis inhibitors in combination with chemotherapeutics and gene/antibody therapy, are additional treatment options. However, as the brain is difficult to access due to the presence of the blood-brain barrier (BBB), none of the above-mentioned therapies have been successful in curing this disease. The lack of knowledge regarding the mechanisms underlying the development and progression of gliomas further adds to the difficulties. Currently, investigations are focused on the development of novel methods for improving the outcome of this disease. However, despite the extensive investigations, $88 \%$ of all glioblastoma multiforme (GBM) patients succumb to the disease within 3 years. GBM remains one of the most challenging malignancies worldwide.
\end{abstract}

\section{Contents}

1. Introduction

2. Current perspectives

Correspondence to: Dr Radovan Komel or Ms. Ivana Jovčevska, Medical Center for Molecular Biology, Institute of Biochemistry, Faculty of Medicine, University of Ljubljana, 2 Vrazov Trg, 1000 Ljubljana, Slovenia

E-mail: radovan.komel@mf.uni-lj.si

E-mail: ivana.jovcevska@mf.uni-lj.si

Key words: glioma, glioblastoma multiforme, nanobodies, cancer stem cells
3. Future possibilities

4. Conclusion

\section{Introduction}

Over 11 million individuals are diagnosed with cancer annually and it was estimated that, by the year 2020, this number will rise to 16 million $(1,2)$. Malignant gliomas are the most common type of primary brain tumors, with an annual incidence of 5/100,000 individuals $(3,4)$. The first glioma classification was proposed by Bailey and Cushing (5). Currently, the classification established by the World Health Organization (WHO) is used (3), which divides gliomas into astrocytomas, oligodendrogliomas, ependymomas and oligo-astrocytomas (mixed gliomas). For further easier classification the tumors may be subdivided into stages, taking into account the size of the tumor, the level of penetration and spread to the lymph nodes or other adjacent or distant organs. Such a system is the TNM staging system, where ' $\mathrm{T}$ ' stands for tumor, ' $\mathrm{N}$ ' for lymph nodes and ' $\mathrm{M}$ ' for metastasis. Thus far, five stages have been described (6) (Table I).

According to the grade of malignancy, oligodendrogliomas and oligoastrocytomas are grade II and grade III. Astrocytomas are subdivided as follows: pilocytic, grade I; diffuse, grade II; anaplastic, grade III; and glioblastoma multiforme (GBM), grade IV $(7,8)$. Glioblastomas account for $60-70 \%$ of all gliomas, anaplastic astrocytomas for $10-15 \%$, anaplastic oligodendrogliomas and anaplastic oligoastrocytomas for $10 \%$ and the remaining 5-20\% belong to less common tumor types, such as anaplastic ependymomas and anaplastic gangliogliomas (4).

The term 'multiforme' reflects the heterogeneity of this type of tumor regarding clinical presentation, pathology, genetic signature and response to treatment (9). GBM may be primary (arising de novo) or secondary (arising from a lower-grade tumor) (10). Primary glioblastomas are more common, usually occur in patients aged $>50$ years and exhibit the following genetic characteristics: deletion of phosphatase and tensin homologue genes on chromosome 10 and p16 deletion and loss of heterozygosity on chromosomes 10q and 17p (11). Secondary glioblastomas are more common among younger patients. They arise as low-grade tumors and, over a few years, they progress to glioblastomas. They are characterized by mutations in the p53 tumor suppressor gene, abnormalities in the p16 and retinoblastoma pathways and aberrations in the DNA copy number. The pathways in the development 
Table I. Cancer staging according to the TNM system.

\begin{tabular}{ll}
\hline Stage & \multicolumn{1}{c}{ Description } \\
\hline 0 & Carcinoma in situ \\
I & Cancer is localized to one part of the body \\
II & Cancer is locally advanced \\
III & Cancer is more advanced \\
IV & Cancer has metastasized (spread to other organs)
\end{tabular}

TNM, tumor-node-metastasis.

of malignant gliomas were described in detail by Wen and Kesari (4) and the major genetic alterations in primary and secondary GBM initiation and progression were described by Li et al (12). As regards secondary glioblastomas, the most common origins of brain metastatic lesions are primary cancers of the lung (65\%) (13), skin (melanomas, 10-40\%) (14), breast and gastrointestinal tract (13). Despite their molecular differences, the two tumor types are morphologically indistinguishable and respond similarly to conventional therapy.

The majority of GBM patients $(87 \%)$ are aged 55-87 years (15). This disease is more common among men compared to women and less common among black compared to Caucasian populations $(4,16)$. Only $\sim 5 \%$ of the patients have a family history of the disease (17) and the only known risk factor is exposure to ionizing radiation (18). Patients suffering from GBM have a median survival of 15 months $(8,19,20)$, whereas those with anaplastic astrocytoma have a median survival of $\leq 3$ years (21). Recurrent gliomas have a worse prognosis, with a median patient survival of 3-6 months (22).

\section{Current perspectives}

Cancer origin. It has been previously hypothesized that the brain does not regenerate; however, recent findings demonstrated that spontaneous tissue repair occurs in patients with inflammatory and degenerative disorders of the central nervous system (CNS), although this regeneration is not sufficient for complete recovery of the CNS (23). Neural stem cells (NSCs) are considered responsible for this phenomenon. NSCs have the ability to self-renew, i.e., produce identical cells, are mitotically active, multipotent and exhibit complex patterns of genetic expression (24-26). Thus far, NSCs have been isolated from the following regions of the mammalian CNS: subventricular zone, hippocampus and dentate gyrus in the temporal lobe (27). CNS NSCs are able to coordinate tissue generation, maintenance and regeneration. It is also believed that they are able to generate new neurons, i.e., are involved in neurogenesis in specialized brain regions, such as the olfactory bulb, the hippocampus and the central canal of the spinal cord (28-30). The origins of brain NSCs are still debated upon.

The mechanisms underlying cancer development in the human body have not been elucidated. Cancer is considered to be a cluster of diseases involving gene rearrangements and amplifications, point mutations, alterations in proto-oncogenes, tumor suppressor genes and DNA repair genes. It is hypothesized that epigenetic alterations, such as DNA methylation and different patterns of histone modifications may also be involved $(1,31,32)$. There are two different potential mechanisms underlying this disease: the stochastic model, in which all the tumor cells are equipotent and, as a result of genetic or epigenetic changes, some may randomly contribute to tumor growth $(33,34)$; and the cancer stem cell (CSC) model, which suggests that only a specific fraction of the tumor cells have the ability to proliferate and give rise to a tumor. There is also the possibility that cancer cells deviate from the hierarchical model into anarchy, as they progress from benign to malignant (35).

One hundred and fifty years ago, Rudolf Virchow, a German pathologist, developed a theory proposing that cancer arises from immature cells $(36,37)$. One hundred years later, Sajiro Makino introduced the term 'cancer stem cells', describing a subpopulation of cells resistant to chemotherapy and with different chromosomal characteristics compared to other cells (38). The CSC field is relatively new and unexplored. Thus far, researchers use the general knowledge on SCs (ability to self renew, multipotency) to identify CSCs. Little is known regarding normal SCs, CSCs and their mutual and distinct characteristics. It has been hypothesized that the accumulation of mutations in the normal SCs over a period of time gives rise to CSCs $(34,39)$. This may be due to the fact that SCs have a longer life compared to differentiated cells; therefore, the probability of inheriting a gained mutation is higher. Another theory is that CSCs arise from a mutated progenitor cell (39).

The function of CSCs has not been elucidated. It remains unknown whether CSCs are the origin of all malignant cells in the body, whether they are responsible for the existence of drug-resistant cancer cells, or whether they initiate metastasis (39). In general, it is hypothesized that chemotherapy eliminates the majority, but not all cancer cells. Those that are spared are the CSCs that reappear more aggressively after the initial treatment (40).

CSCs may be distinguished from normal SCs by their ability to efflux the Hoechst 33342 DNA dye $(26,36,37)$. The blue fluorescent Hoechst 33342 dye is a cell-permeable bis-benzimidazole derivative that binds to the minor DNA groove. Following excitation, the dye emission may be measured in the blue $(450 \mathrm{~nm})$ and the red $(675 \mathrm{~nm})$ spectrum. The dye enters viable cells and it is pumped out by the ATP-binding cassette $(\mathrm{ABC})$ transporters of the cell membrane $(41,42)$. CSCs are overexpressing ABCG2, an ABC protein transporter capable of effluxing several hydrophobic compounds, including the Hoechst 33342 dye and chemotherapeutic drugs, such as topotecan and doxorubicin (43). The rapid efflux of the chemotherapeutic agents may be the reason for the resistance of CSCs to treatment. As a result of the rapid efflux of the dye, a side population (SP) of weakly stained cells may be observed by flow cytometric analysis (26). Goodell et al (44) previously described a technique for isolating the SP: the measurement of the emission of Hoechst fluorescence in the blue and red spectrum points to a small cell population (accounting for $0.1 \%$ of all cells), which expresses stem cell markers. Thus far, SPs have been identified in glioblastoma, lung and breast cancers (45) and in normal tissues, including lung, liver, brain and skin, in mouse and human models (46-49). The dye may be kept inside the cell with the use of verapamil, an L-type calcium channel 
blocking agent. Blocking the calcium channels inhibits the Hoechst dye efflux and the size of the cell population suspected to be CSCs may be determined by differential analysis $(36,37)$. It should be considered that the dye binds to the cell DNA, which renders it potentially mutagenic and carcinogenic and its toxicity increases with longer exposure.

Rhodamine 123 may be used instead of the Hoechst 33342 dye, since it was shown to be non-toxic, even at higher concentrations (50). Rhodamine 123 is a green fluorescent cell-permeable dye that binds to the mitochondrial membranes. Rhodamine 123 is also actively pumped out by the ABC transporters. The Rhodamine 123 and Hoechst 33342 dyes were compared and were shown to yield the same percentage of stained cells and identical proliferative abilities in vitro and expression of stem cell markers in vivo (51).

CSC markers. Different cell markers may be used for the identification or purification of the CSC population. Certain markers, such as $\mathrm{CD} 133^{+}$, which is used for the identification of brain tumors and lung cancer, and $\mathrm{CD}_{3} 4^{+}$, which is used for hematological malignancies, were described by Vermeulen et al (34).

CD133 (Prominin-1) is expressed in hematopoietic stem cells, endothelial progenitor cells, neuronal and glial stem cells $(40,52)$. In addition, it was previously shown to be present in subpopulations of brain, lung, melanoma and other solid tumor cancer cells, which led to the hypothesis that it may be used as a CSC marker (53-55). A monoclonal antibody (MAb) that recognizes the AC133 antigen, a glycosylation-dependent epitope of CD133, was previously developed (56). A complementary DNA cloning and tissue distribution study demonstrated that AC133 is an 865-amino acid (aa) single-chain polypeptide with a molecular weight of $120 \mathrm{kDa}$ (56-58). The AC133 antigen expression was restricted to $\mathrm{CD} 34^{+}$hematopoietic stem cells, unlike AC133 mRNA, which is widespread and detected in the brain, heart, liver and lung (57). A multiple tissue expression array revealed the expression of human CD133 mRNA in the kidney, trachea, pancreas and testis (59), which was consistent with findings in mouse models (60). Of note, AC133 is not a synonym for CD133. Investigations on the transcriptional regulation of the human $A C 133$ gene revealed the presence of 9 different $5^{\prime}$ untranslated exon regions, resulting in at least 7 alternatively spliced forms of AC133 mRNA, which are expressed in a tissue-dependent manner (61). As the CD133 marker is detected by its glycosylated epitope, AC133 may be considered as a better stem cell marker.

CSCs expressing CD133 were isolated from several human brain tumors, including glioblastomas $(27,40,55,62)$. These cells are able to differentiate in neurons, astrocytes and oligodendrocytes in vitro and may recapitulate the original tumor phenotype in vivo, unlike their CD133- counterparts (58). It was demonstrated that the expression of CD133 is higher in recurrent glioblastoma tissues compared to that in primary tumors. However, it was revealed that cells not expressing the CD133 marker may also be tumorigenic. It was reported that CD133- cells derived from 6 patients were proven to be tumorigenic when inserted into mouse brains. For three of those, the resulting tumors were $\mathrm{CD} 133^{+}(36,37)$.

Diagnosis. Gliomas are difficult to diagnose early, as the presenting symptoms may be common (e.g., headache, seizures, confusion, memory loss and personality changes), although problems with speech or motor function may appear but go unnoticed (63). The diagnosis is usually confirmed by magnetic resonance imaging (MRI) or computed tomography and usually appear as a mass with surrounding edema. Functional MRI may be used to determine the involvement of speech and motor areas. For measuring the metabolite level and distinguishing a tumor from necrotic areas or benign lesions, proton magnetic resonance spectroscopy (MRS) may be used (4). In vivo MRS detects metabolites, such as $\mathrm{N}$-acetylaspartate (NAA), choline (Cho), creatine $(\mathrm{Cr})$, myo-inositol, lactate and lipids, by measuring the spectra of specific isotopes, such as ${ }^{1} \mathrm{H},{ }^{13} \mathrm{C}$ or ${ }^{31} \mathrm{P}(64)$. Since the metabolite level varies in different parts of the brain and among age groups, it was suggested to measure metabolite ratios, such as Cho/Cr and NAA/Cr, rather than measuring absolute metabolite concentrations $(65,66)$. However, as these methods may be costly, there is a need for a novel diagnostic method. For this purpose, microRNAs (miRNAs) may be used. miRNAs are small non-coding molecules that regulate gene expression. It was hypothesized that extracellular miRNAs found in the plasma or serum may be used as potential biomarkers for cancer detection. Their key properties are that their expression is tissue-specific, their expression levels are stable and may be detected in the plasma, but are altered in the presence of cancer or other disease (67-69). Wang et al (70) reported their results on miRNA detection in plasma samples (blood-based biomarkers) obtained from patients and healthy individuals. The results of that study appeared promising regarding the use of miR-21, which was upregulated, and miR-128 and $-342-3 p$, which were downregulated in glioma patients. These three miRNAs may be used to discriminate between GBM and other brain tumors.

Treatment. The treatment of GBM currently involves surgery followed by external-beam radiation and concomitant temozolomide (TMZ) chemotherapy followed by an additional 6 cycles of TMZ administration (71). TMZ is a small alkylating agent (MW 194.15) that kills cancer cells by forming $\mathrm{O}^{6}$-methylguanine in DNA, which miss-pairs with thymine $(\mathrm{T})$ during the next DNA replication cycle. The same mismatch occurs in the successive replication cycles, eventually leading to cell death (40). In rat models, it was demonstrated that TMZ crosses the blood brain barrier (BBB) and achieves higher intratumoral concentrations compared to several other cytotoxic therapies (72). Surgery may be complicated, due to the infiltrative nature of the tumor, although it may be useful as a biopsy providing tissue for diagnosis and further research. Radiation was shown to prolong survival up to 12 months $(4,71,73)$; however, in $90 \%$ of the cases tumors reoccur in the primary site. Chemotherapy is used in combination with radiation and may help prolong patient survival. Combining an angiogenesis inhibitor (such as bevacizumab) with a chemotherapeutic agent (irinotecan) may achieve better results (16,74). In theory, a cytotoxic and an anti-angiogenic agent may complement each other and be more effective in decreasing tumor cell proliferation, inducing cancer cell death and reducing tumor-associated inflammation (72).

The WHO grade IV glioblastoma treatment consists of extensive surgical resection, radiotherapy and TMZ. For recur- 
rent gliomas, the treatment involves reoperation, conventional chemotherapy, administration of bevacizumab and irinotecan, as well as experimental procedures (4).

The options for the treatment of GBM are limited due to the presence of the $\mathrm{BBB}$, which prevents molecules $>500 \mathrm{Da}$ (15) from entering the brain. The BBB is a selective physical barrier, as the tight junctions between the adjacent endothelial cells do not allow for the normal, paracellular transport, but force molecules into a transcellular transport. Small molecules, such as $\mathrm{O}_{2}, \mathrm{CO}_{2}$ and ethanol may diffuse freely through the membrane (75-77). The presence of specific transport systems on the membrane surface enables nutrients to enter the brain, but prevents potentially toxic substances from harming the CNS. Large molecules, such as peptides and proteins, are not able to enter the brain, unless there is a strictly regulated receptor-mediated or adsorption-mediated transcytosis (78). The BBB has a protective role: it mediates the efflux of waste products, maintains the ionic concentrations, which may change significantly following a meal and cause a disruption of normal brain function, and it separates the pools of the neurotransmitters that act centrally and peripherally. Overall, the BBB maintains the homeostasis of the CNS $(79,80)$. Considering the limited penetration in the brain, alternative drug-delivery strategies are required for the more effective treatment of gliomas.

Determining the differences between normal SCs and CSCs is important for further investigations as well as for understanding the failure of current treatment. As the known treatments do not offer a cure for glioma, the cellular and molecular characteristics of these two types of cells require further elucidation. Such knowledge may provide better insight into drug function and targeting, tumor reoccurrence and drug resistance.

\section{Future possibilities}

Immunotherapeutic approaches. Examples of experimental procedures were described by Hickey et al (81). Two types of immunotherapy were outlined, passive and active. Passive immunotherapy is the adoptive transfer of ex vivo activated cytotoxic effector cells to a patient, whereas the immunization of patients upon activation of endogenous immune cells is defined as active immunotherapy (81).

There have already been some attempts for active immunotherapeutic approaches (81). However, the outcomes of those trials remain a subject under discussion. For better results and understanding of the experiments, data such as previous medical information, disease treatment, patient response, previously administered chemotherapeutic agents and radiation courses have to be generated. Overall, there have been advances in this field, although further investigations are required for the immunotherapeutic approaches to be established as a safe treatment option for glioma patients.

Newly acquired knowledge regarding the pathology underlying glioma may help develop novel, more successful treatment strategies. The angiogenesis hypothesis, i.e., the development of tumor-associated blood vessels that are required for solid tumor growth, was proposed by Folkman (82). As gliomas are highly vascular tumors expressing vascular endothelial growth factor (VEGF), targeting the vascular endothelium may be a viable therapeutic option. In gliomas, the level of VEGF expression correlates with blood vessel density, malignancy grade and prognosis (83). Based on the fact that solid tumors require the generation of new blood vessels in order to develop, new therapies are based on anti-angiogenic strategies $(84,85)$.

Malignant gliomas are among the most highly vascular human tumors (86). A combination of bevacizumab and irinotecan was shown to achieve a response rate of 57-63\% among glioma patients $(87,88)$. Bevacizumab is a humanized immunoglobulin G1 MAb that affects the vascular endothelium and was proven to be effective in metastatic colorectal, breast and lung cancers $(89,90)$. Irinotecan is a topoisomerase I inhibitor with a mechanism of action different from that of alkylating agents, such as TMZ, which forms $\mathrm{O}^{6}$-methylguanine in the DNA helix that miss-pairs with $\mathrm{T}$ in the next DNA replication cycle. Its use was shown to achieve a response rate of $0-17 \%$ in patients in several clinical trials $(91,92)$. Vredenburgh et al $(87)$ performed a phase II clinical trial of bevacizumab and irinotecan for patients with recurrent glioblastoma, which reported an encouraging preliminary response rate of $43 \%$. However, despite the promising initial results, there were 2 treatment-related deaths and the treatment was discontinued in some of the patients due to complications. Bevacizumab is generally known to be well-tolerated, although the main concern is its contribution to fatigue and hemorrhage, which has not been precisely defined (83). An advantage of this drug may be its ability to decrease peritumoral edema. In addition, the authors of that study observed a reduction in the steroid dose in $33 \%$ of patients who had already received bevacizumab, which may suggest its function as a corticosteroid-sparing agent.

The first clinical evidence for the use of bevacizumab and irinotecan in the treatment of recurrent malignant glioma was published by Stark-Vance (93). The results of that study were supported by those reported by Vredenburgh et al (87) and Pope et al (94). It was reported that $77 \%$ of the treated patients exhibited a partial response and $23 \%$ had stable disease (16). It was also reported that $9.8 \%$ of the patients experienced deep vein thrombosis, 2 patients developed pulmonary embolism, $16.4 \%$ experienced extreme fatigue and a further $9.8 \%$ had intracerebral hemorrhage and bone marrow toxicity as a result of the treatment. However, there were no reported deaths as a direct result of the chemotherapy. The primary goal of bevacizumab is blocking tumor endothelial cell proliferation and normalizing the existing vasculature (95), which achieves promising short-term results in decreasing the tumor mass. The complications that may arise due to the impact on cell migration have not yet been determined.

Gene therapy. Gene therapy, i.e., the treatment of the cause rather than the symptoms of a disease, is considered a revolution in medicine (96). The first human gene therapy trial was performed in 1990 on a female patient born with a defective adenosine deaminase gene (11). That trial had a positive outcome, resulting in the emergence of a variety of human gene treatment protocols worldwide. Thus far, the cancer protocol is the most popular (848 patients), followed by AIDS (372 patients) and cystic fibrosis (152 patients) (11). Viral vectors used in gene therapy are retroviruses, adenoviruses and adeno-associated viruses (AAVs). However, they all have their limitations: the cells need to be 
undergoing division (retroviruses), adenoviruses may trigger an immune response and, as regards AAVs, there is no efficient production method. Non-viral vectors, such as DNA/liposomes, were proven to be non-infectious and non-immunogenic and, therefore, are safer compared to the viral vectors. For malignant glioma, there are two approaches for gene therapy, the suicide gene therapy, which uses the herpes simplex thymidine kinase gene and ganciclovir, and the immune gene therapy, which uses cytokine genes. In the suicide gene therapy, a significant reduction in the tumor size was observed (11).

The Ab-based gene therapy is still 'under construction'. Antibodies (Abs) have an advantage over other therapeutic agents, as they are of small size and may target the tumor directly. Another benefit of using Abs is their rapid clearance from the human body, which makes them less immunogenic and well-tolerated (85). However, solid tumors are relatively resistant to Ab-based therapies, with only $0.001-0.01 \%$ of the injected $\mathrm{Ab}$ dose incorporated per gram of solid tumor (97).

Recombinant Abs have been generated in order to improve the outcomes of this type of therapy. The use of bispecific Abs (bsAbs) is one of the trial approaches. bsAbs contain two distinct binding specificities (98) and may enhance the effector activity of lymphocytes or phagocytes by binding to cell activation molecules with one domain, while binding to specific surface antigens on the target molecules with the other domain (98). However, current problems in this field are the low retention time, which leads to the need for frequent $\mathrm{Ab}$ administration, large scale production and toxicity as a result of acute cytokine release and biodistribution (85). A somewhat different approach is the use of bispecific diabodies, which are recombinant constructs containing two single-chain Fv fragments. They are focused on T-cell recruitment, with one Fv module targeted to CD3 on T cells and the other targeted to a cancer marker (99). Bispecific diabodies have two advantages over bsAbs: first, as a result of the absence of the Fc domain, they only activate $\mathrm{T}$ cells when cross-linked to the target cells and, second, the anti-bispecific response in the host is minimized due to the small size.

Nanobodies. A slightly different aspect in Ab-based gene therapy is the use of nanobodies. Nanobodies are naturally occurring single-domain Abs, which may be fully functional, even in the absence of a light chain. The name originates from the small size of their antigen-binding fragment, which is only $15 \mathrm{kDa}(100)$. Nanobodies are stable and robust and their small size may enable their penetration into tissues which are currently inaccessible. Their small size makes them suitable for targeted therapy and discovery of new biomarkers, which is also performed in our laboratory as part of the Crossborder Cooperation Programme Italy-Slovenia 2007-2013 (GLIOMA project). In addition, the nanobodies derived from camelids are homologous with the human VH3 gene family and should therefore be less immunogenic (101). However, further investigations are required to determine their affinity, stability and pharmacokinetic properties.

In the treatment of cancer, the optimal method would be to target the drug directly to the tumor, which may make the treatment more effective, while significantly reducing the side effects and damages to healthy tissues. Song et al (102) introduced the use of small interfering RNAs (siRNAs) for
mRNA degradation. siRNAs may be delivered to the cells through antibody-mediated endocytosis. This method was not proven to be particularly effective, although it has advantages such as non-activation of the innate immune system and lack of infection risk (103). Additional problems may arise due to the fact that cancers are heterogeneous. It remains to be investigated whether targeting is successful for all cancer cells in the body or for just a specific group (primary cancer and/or metastasis).

\section{Conclusion}

Brain tumors represent a wide field of investigation. Glioma is an 'intelligent' tumor that manages to escape conventional radiation and chemotherapy treatment methods, thus being fatal to the majority of the patients. Experimental procedures, gene/antibody therapy and current clinical trials may lead to improvements in the outcome of these patients in the future. Despite the extensive investigations, a cure for this disease is currently not available. Information regarding the tumor biology is available in the literature, although it is clearly insufficient. A thorough and detailed understanding of the mechanisms of metastasis, drug resistance and tumor occurrence is required for the treatments to be more successful and treatment challenges, such as targeting the tumor and sparing the normal and healthy tissue from toxic substances and, particularly, the BBB, need to be overcome.

\section{Acknowledgements}

The authors would like to thank the Crossborder Cooperation Programme Italy-Slovenia 2007-2013 (GLIOMA project) and the Slovene Human Resources Development and Scholarship Fund (JR. 113) for the financial support.

\section{References}

1. Bhatt AN, Mathur R, Farooque A, Verma A and Dwarakanath BS: Cancer biomarkers - current perspectives. Indian J Med Res 132: 129-149, 2010.

2. Cho WC: Contribution of oncoproteomics to cancer biomarker discovery. Mol Cancer 6: 25, 2007.

3. Louis DN, Ohgaki H, Wiestler OD, et al: The 2007 WHO classification of tumours of the central nervous system. Acta Neuropathol 114: 97-109, 2007.

4. Wen PY and Kesari S: Malignant gliomas in adults. N Engl J Med 359: 492-507, 2008.

5. Bailey $\mathrm{P}$ and Cushing H: A Classification of the Tumors of the Glioma Group on a Histogenetic Basis with a Correlated Study of Prognosis. JB Lippincott \& Co., Philadelphia, PA, 1926.

6. Galon J, Pages F, Marincola FM, et al: Cancer classification using the Immunoscore: a worldwide task force. J Transl Med 10: 205, 2012

7. Louis DN, Holland EC and Cairncross JG: Glioma classification: a molecular reappraisal. Am J Pathol 159: 779-786, 2001.

8. Okada H, Kohanbash G, Zhu X, et al: Immunotherapeutic approaches for glioma. Crit Rev Immunol 29: 1-42, 2009.

9. Iacob G and Dinca EB: Current data and strategy in glioblastoma multiforme. J Med Life 2: 386-393, 2009.

10. Bralten LB and French PJ: Genetic alterations in glioma. Cancers 3: 1129-1140, 2011.

11. Yoshida J: Molecular neurosurgery using gene therapy to treat malignant glioma. Nagoya J Med Sci 59: 97-105, 1996.

12. Li J, Di C, Mattox AK, Wu L and Adamson DC: The future role of personalized medicine in the treatment of glioblastoma multiforme. Pharmgenomics Pers Med 3: 111-127, 2010.

13. Chi A and Komaki R: Treatment of brain metastasis from lung cancer. Cancers 2: 2100-2137, 2010. 
14. Mathieu D, Kondziolka D, Cooper PB, et al: Gamma knife radiosurgery for malignant melanoma brain metastases. Clin Neurosurg 54: 241-247, 2007

15. Daga A, Bottino C, Castriconi R, Gangemi R and Ferrini S: New perspectives in glioma immunotherapy. Curr Pharm Des 17: 2439-2467, 2011

16. Ali SA, McHayleh WM, Ahmad A, et al: Bevacizumab and irinotecan therapy in glioblastoma multiforme: a series of 13 cases. J Neurosurg 109: 268-272, 2008.

17. Farrell CJ and Plotkin SR: Genetic causes of brain tumors: neurofibromatosis, tuberous sclerosis, von Hippel-Lindau, and other syndromes. Neurol Clin 25: 925-946, viii, 2007.

18. Fisher JL, Schwartzbaum JA, Wrensch M and Wiemels JL: Epidemiology of brain tumors. Neurol Clin 25: 867-890, vii, 2007.

19. Chen J, Li Y, Yu TS, et al: A restricted cell population propagates glioblastoma growth after chemotherapy. Nature 488: 522-526, 2012.

20. Westermark B: Glioblastoma - a moving target. Ups J Med Sci 117: 251-256, 2012.

21. Van Gool S, Maes W, Ardon H, Verschuere T, Van Cauter S and De Vleeschouwer S: Dendritic cell therapy of high-grade gliomas. Brain Pathol 19: 694-712, 2009.

22. Gruber ML and Buster WP: Temozolomide in combination with irinotecan for treatment of recurrent malignant glioma. Am J Clin Oncol 27: 33-38, 2004.

23. Pluchino S, Zanotti L, Deleidi M and Martino G: Neural stem cells and their use as therapeutic tool in neurological disorders. Brain Res Brain Res Rev 48: 211-219, 2005.

24. Goldman S: Glia as neural progenitor cells. Trends Neurosci 26 : 590-596, 2003

25. Ivanova NB, Dimos JT, Schaniel C, Hackney JA, Moore KA and Lemischka IR: A stem cell molecular signature. Science 298 601-604, 2002

26. Hadnagy A, Gaboury L, Beaulieu R and Balicki D: SP analysis may be used to identify cancer stem cell populations. Exp Cell Res 312: 3701-3710, 2006.

27. Singh SK, Clarke ID, Terasaki M, et al: Identification of a cancer stem cell in human brain tumors. Cancer Res 63: 5821-5828, 2003.

28. Altman J: Autoradiographic and histological studies of postnatal neurogenesis. 3. Dating the time of production and onset of differentiation of cerebellar microneurons in rats. J Comp Neurol 136: 269-293, 1969

29. Corotto FS, Henegar JA and Maruniak JA: Neurogenesis persists in the subependymal layer of the adult mouse brain. Neurosci Lett 149: 111-114, 1993.

30. Eriksson PS, Perfilieva E, Bjork-Eriksson T, et al: Neurogenesis in the adult human hippocampus. Nat Med 4: 1313-1317, 1998.

31. Baylin SB and Ohm JE: Epigenetic gene silencing in cancer a mechanism for early oncogenic pathway addiction? Nat Rev Cancer 6: 107-116, 2006.

32. Jaenisch R and Bird A: Epigenetic regulation of gene expression: how the genome integrates intrinsic and environmental signals. Nat Genet 33 Suppl: 245-254, 2003

33. Driessens G, Beck B, Caauwe A, Simons BD and Blanpain C: Defining the mode of tumour growth by clonal analysis. Nature 488: 527-530, 2012.

34. Vermeulen L, Sprick MR, Kemper K, Stassi G and Medema JP Cancer stem cells - old concepts, new insights. Cell Death Differ 15: 947-958, 2008

35. Gilbertson RJ and Graham TA: Cancer: Resolving the stem-cell debate. Nature 488: 462-463, 2012.

36. Ailles LE and Weissman IL: Cancer stem cells in solid tumors Curr Opin Biotechnol 18: 460-466, 2007.

37. Welte Y, Adjaye J, Lehrach HR and Regenbrecht CR: Cancer stem cells in solid tumors: elusive or illusive? Cell Commun Signal 8: 6, 2010.

38. Makino S: The role of tumor stem-cells in regrowth of the tumor following drastic applications. Acta Unio Int Contra Cancrum 15 (Suppl 1): 196-198, 1959.

39. Jordan CT, Guzman ML and Noble M: Cancer stem cells. N Engl J Med 355: 1253-1261, 2006

40. Liu G, Yuan X, Zeng Z, et al: Analysis of gene expression and chemoresistance of $\mathrm{CD} 133^{+}$cancer stem cells in glioblastoma. Mol Cancer 5: 67, 2006.

41. Albini A and Sporn MB: The tumour microenvironment as a target for chemoprevention. Nat Rev Cancer 7: 139-147, 2007.

42. Calabrese C, Poppleton H, Kocak M, et al: A perivascular niche for brain tumor stem cells. Cancer Cell 11: 69-82, 2007.
43. Crea F, Danesi R and Farrar WL: Cancer stem cell epigenetics and chemoresistance. Epigenomics 1: 63-79, 2009.

44. Goodell MA, Brose K, Paradis G, Conner AS and Mulligan RC: Isolation and functional properties of murine hematopoietic stem cells that are replicating in vivo. J Exp Med 183: 1797-1806, 1996.

45. Hirschmann-Jax C, Foster AE, Wulf GG, et al: A distinct 'side population' of cells with high drug efflux capacity in human tumor cells. Proc Natl Acad Sci USA 101: 14228-14233, 2004.

46. Martin CM, Meeson AP, Robertson SM, et al: Persistent expression of the ATP-binding cassette transporter, Abcg2, identifies cardiac SP cells in the developing and adult heart. Dev Biol 265: 262-275, 2004

47. Shimano K, Satake M, Okaya A, et al: Hepatic oval cells have the side population phenotype defined by expression of ATP-binding cassette transporter ABCG2/BCRP1. Am J Pathol 163: 3-9, 2003.

48. Umemoto T, Yamato M, Nishida K, Yang J, Tano Y and Okano T: Limbal epithelial side-population cells have stem cell-like properties, including quiescent state. Stem Cells 24: 86-94, 2006.

49. Yano S, Ito Y, Fujimoto M, Hamazaki TS, Tamaki K and Okochi H: Characterization and localization of side population cells in mouse skin. Stem Cells 23: 834-841, 2005.

50. Ribou AC, Vigo J, Kohen E and Salmon JM: Microfluorometric study of oxygen dependence of (1"-pyrene butyl)-2-rhodamine ester probe in mitochondria of living cells. J Photochem Photobiol B 70: 107-115, 2003.

51. Liu WH, Qian NS, Li R and Dou KF: Replacing Hoechst33342 with rhodamine123 in isolation of cancer stem-like cells from the MHCC97 cell line. Toxicol In Vitro 24: 538-545, 2010.

52. Pfenninger CV, Roschupkina T, Hertwig F, et al: CD133 is not present on neurogenic astrocytes in the adult subventricular zone, but on embryonic neural stem cells, ependymal cells, and glioblastoma cells. Cancer Res 67: 5727-5736, 2007.

53. Ieta $\mathrm{K}$, Tanaka $\mathrm{F}$, Haraguchi $\mathrm{N}$, et al: Biological and genetic characteristics of tumor-initiating cells in colon cancer. Ann Surg Oncol 15: 638-648, 2008.

54. Monzani E, Facchetti F, Galmozzi E, et al: Melanoma contains CD133 and ABCG2 positive cells with enhanced tumourigenic potential. Eur J Cancer 43: 935-946, 2007.

55. Singh SK, Hawkins C, Clarke ID, et al: Identification of human brain tumour initiating cells. Nature 432: 396-401, 2004.

56. Yin AH, Miraglia S, Zanjani ED, et al: AC133, a novel marker for human hematopoietic stem and progenitor cells. Blood 90: 5002-5012, 1997

57. Miraglia S, Godfrey W, Yin AH, et al: A novel five-transmembrane hematopoietic stem cell antigen: isolation, characterization, and molecular cloning. Blood 90: 5013-5021, 1997.

58. Mizrak D, Brittan M and Alison M: CD133: molecule of the moment. J Pathol 214: 3-9, 2008.

59. Florek M, Haase M, Marzesco AM, et al: Prominin-1/CD133, a neural and hematopoietic stem cell marker, is expressed in adult human differentiated cells and certain types of kidney cancer. Cell Tissue Res 319: 15-26, 2005

60. Weigmann A, Corbeil D, Hellwig A and Huttner WB: Prominin, a novel microvilli-specific polytopic membrane protein of the apical surface of epithelial cells, is targeted to plasmalemmal protrusions of non-epithelial cells. Proc Natl Acad Sci USA 94: 12425-12430, 1997.

61. Shmelkov SV, Jun L, St Clair R, et al: Alternative promoters regulate transcription of the gene that encodes stem cell surface protein AC133. Blood 103: 2055-2061, 2004.

62. Bao S, Wu Q, McLendon RE, et al: Glioma stem cells promote radioresistance by preferential activation of the DNA damage response. Nature 444: 756-760, 2006.

63. Yamanaka R: Cell- and peptide-based immunotherapeutic approaches for glioma. Trends Mol Med 14: 228-235, 2008.

64. Bulik M, Jancalek R, Vanicek J, Skoch A and Mechl M: Potential of MR spectroscopy for assessment of glioma grading. Clin Neurol Neurosurg 115: 146-153, 2013

65. Komoroski RA, Heimberg C, Cardwell D and Karson CN: Effects of gender and region on proton MRS of normal human brain. Magn Reson Imaging 17: 427-433, 1999.

66. Li BS, Wang H and Gonen O: Metabolite ratios to assumed stable creatine level may confound the quantification of proton brain MR spectroscopy. Magn Reson Imaging 21: 923-928, 2003.

67. Calin GA and Croce CM: MicroRNA signatures in human cancers. Nat Rev Cancer 6: 857-866, 2006.

68. Lu J, Getz G, Miska EA, et al: MicroRNA expression profiles classify human cancers. Nature 435: 834-838, 2005. 
69. Mitchell PS, Parkin RK, Kroh EM, et al: Circulating microRNAs as stable blood-based markers for cancer detection. Proc Natl Acad Sci USA 105: 10513-10518, 2008.

70. Wang Q, Li P, Li A, et al: Plasma specific miRNAs as predictive biomarkers for diagnosis and prognosis of glioma. J Exp Clin Cancer Res 31: 97, 2012.

71. Stupp R, Mason WP, van den Bent MJ, et al: Radiotherapy plus concomitant and adjuvant temozolomide for glioblastoma. N Engl J Med 352: 987-996, 2005.

72. Grossman R, Rudek MA, Brastianos H, et al: The impact of bevacizumab on temozolomide concentrations in intracranial U87 gliomas. Cancer Chemother Pharmacol 70: 129-139, 2012.

73. Walker MD, Alexander E Jr., Hunt WE, et al: Evaluation of $\mathrm{BCNU}$ and/or radiotherapy in the treatment of anaplastic gliomas. A cooperative clinical trial. J Neurosurg 49: 333-343, 1978.

74. Reardon DA, Quinn JA, Rich JN, et al: Phase I trial of irinotecan plus temozolomide in adults with recurrent malignant glioma. Cancer 104: 1478-1486, 2005.

75. Abbott NJ, Ronnback L and Hansson E: Astrocyte-endothelial interactions at the blood-brain barrier. Nat Rev Neurosci 7: 41-53, 2006.

76. Anderson CM and Nedergaard M: Astrocyte-mediated control of cerebral microcirculation. Trends Neurosci 26: 340-345, 2003.

77. Nedergaard M, Ransom B and Goldman SA: New roles for astrocytes: redefining the functional architecture of the brain. Trends Neurosci 26: 523-530, 2003.

78. Pardridge WM: Blood-brain barrier drug targeting: the future of brain drug development. Mol Interv 3: 90-105, 51, 2003.

79. Abbott NJ: Evidence for bulk flow of brain interstitial fluid: significance for physiology and pathology. Neurochem Int 45 545-552, 2004

80. Cserr HF and Bundgaard M: Blood-brain interfaces in vertebrates: a comparative approach. Am J Physiol 246: R277-R288, 1984.

81. Hickey MJ, Malone CC, Erickson KL, et al: Cellular and vaccine therapeutic approaches for gliomas. J Transl Med 8: 100, 2010.

82. Folkman J: Tumor angiogenesis: therapeutic implications. N Engl J Med 285: 1182-1186, 1971.

83. Norden AD, Young GS, Setayesh K, et al: Bevacizumab for recurrent malignant gliomas: efficacy, toxicity, and patterns of recurrence. Neurology 70: 779-787, 2008.

84. Afanasieva TA, Wittmer M, Vitaliti A, Ajmo M, Neri D and Klemenz R: Single-chain antibody and its derivatives directed against vascular endothelial growth factor: application for antiangiogenic gene therapy. Gene Ther 10: 1850-1859, 2003.

85. Sanz L, Blanco B and Alvarez-Vallina L: Antibodies and gene therapy: teaching old 'magic bullets' new tricks. Trends Immunol 25: 85-91, 2004

86. Furnari FB, Fenton T, Bachoo RM, et al: Malignant astrocytic glioma: genetics, biology, and paths to treatment. Genes Dev 21: 2683-2710, 2007.
87. Vredenburgh JJ, Desjardins A, Herndon JE II, et al: Phase II trial of bevacizumab and irinotecan in recurrent malignant glioma. Clin Cancer Res 13: 1253-1259, 2007.

88. Vredenburgh JJ, Desjardins A, Herndon JE II, et al: Bevacizumab plus irinotecan in recurrent glioblastoma multiforme. J Clin Oncol 25: 4722-4729, 2007.

89. Hurwitz H, Fehrenbacher L, Novotny W, et al: Bevacizumab plus irinotecan, fluorouracil, and leucovorin for metastatic colorectal cancer. N Engl J Med 350: 2335-2342, 2004.

90. Johnson DH, Fehrenbacher L, Novotny WF, et al: Randomized phase II trial comparing bevacizumab plus carboplatin and paclitaxel with carboplatin and paclitaxel alone in previously untreated locally advanced or metastatic non-small-cell lung cancer. J Clin Oncol 22: 2184-2191, 2004.

91. Friedman HS, Petros WP, Friedman AH, et al: Irinotecan therapy in adults with recurrent or progressive malignant glioma. J Clin Oncol 17: 1516-1525, 1999.

92. Prados MD, Lamborn K, Yung WK, et al: A phase 2 trial of irinotecan (CPT-11) in patients with recurrent malignant glioma: a North American Brain Tumor Consortium study. Neuro Oncol 8: 189-193, 2006.

93. Stark-Vance V: Bevacizumab and CPT-11 in the treatment of relapsed malignant glioma. Neuro Oncol 7: 369, 2005.

94. Pope WB, Lai A, Nghiemphu P, Mischel P and Cloughesy TF MRI in patients with high-grade gliomas treated with bevacizumab and chemotherapy. Neurology 66: 1258-1260, 2006.

95. Narayana A, Kelly P, Golfinos J, et al: Antiangiogenic therapy using bevacizumab in recurrent high-grade glioma: impact on local control and patient survival. J Neurosurg 110: 173-180, 2009.

96. Mountain A: Gene therapy: the first decade. Trends Biotechnol 18: 119-128, 2000.

97. Carter P: Improving the efficacy of antibody-based cancer therapies. Nat Rev Cancer 1: 118-129, 2001.

98. Segal DM, Weiner GJ and Weiner LM: Bispecific antibodies in cancer therapy. Curr Opin Immunol 11: 558-562, 1999.

99. Hudson PJ: Recombinant antibody constructs in cancer therapy. Curr Opin Immunol 11: 548-557, 1999.

100. Cortez-Retamozo V, Backmann N, Senter PD, et al: Efficient cancer therapy with a nanobody-based conjugate. Cancer Res 64: 2853-2857, 2004

101. Vu KB, Ghahroudi MA, Wyns L and Muyldermans S: Comparison of llama VH sequences from conventional and heavy chain antibodies. Mol Immunol 34: 1121-1131, 1997.

102. Song E, Zhu P, Lee SK, et al: Antibody mediated in vivo delivery of small interfering RNAs via cell-surface receptors. Nat Biotechnol 23: 709-717, 2005.

103. Sioud M: Induction of inflammatory cytokines and interferon responses by double-stranded and single-stranded siRNAs is sequence-dependent and requires endosomal localization. J Mol Biol 348: 1079-1090, 2005. 